\title{
ALDH1 might influence the metastatic capability of HeLa cells
}

\author{
Tingting Yao ${ }^{1,2} \cdot$ Rongbiao $\mathrm{Lu}^{3} \cdot$ Yiqing $\mathrm{Li}^{4}$ • Yongpai Peng ${ }^{1}$ • \\ Miao Ding ${ }^{1} \cdot$ Xiaofei Xie ${ }^{1} \cdot$ Zhongqiu Lin $^{1}$
}

Received: 28 June 2014 / Accepted: 25 March 2015 /Published online: 13 April 2015

(C) The Author(s) 2015. This article is published with open access at Springerlink.com

\begin{abstract}
Recent data suggest that tumor persistence and recurrence could be caused by the presence of cancer stem cells (CSCs). Aldehyde dehydrogenase 1 (ALDH1) has been implicated in cancer pathogenesis and used as a CSC marker. We previously reported that cervical carcinoma contains a small subpopulation of cells expressing ALDH1 [1]. In this study, we used small interfering RNA to suppress ALDH1 expression and introduced an ALDH1 reporting vector into HeLa cells followed by various in vitro assays. We showed that knockdown of ALDH1 expression reduced the cell migration ability of HeLa cells, whereas augmented expression of ALDH1 increased cell migration. However, there was no difference in the cellular proliferation, apoptosis, cell cycle, and invasion. These results indicate that ALDH1 is directly involved in HeLa migration.
\end{abstract}

Keywords ALDH1 $\cdot$ siRNA $\cdot$ Recombinant plasmid . Cervical cancer $\cdot$ HeLa

Tingting Yao

yaoyaotingting@msn.com

Zhongqiu Lin

linzhongqiu@hotmail.com

1 Department of Gynecological Oncology, Sun Yat-sen Memorial Hospital, Sun Yat-sen University, 107 Yan Jiang West Road, Guangzhou 510120, People's Republic of China

2 Key Laboratory of Malignant Tumor Gene Regulation and Target Therapy of Guangdong Higher Education Institute, Sun Yat-sen University, 107 Yan Jiang West Road, Guangzhou 510120, People's Republic of China

3 Department of Dermatology, Third Affiliated Hospital, Sun Yat-sen University, Guangzhou, Guangdong, China

4 Department of Hematology, Sun Yat-Sen Memorial Hospital of Sun Yat-Sen University, Guangzhou, China

\section{Introduction}

Cervical cancer is the third most common cancer in women worldwide and the most frequently occurring cancer of the female reproductive tract [2]. HPV infection has been shown to play a critical role in its etiology; however, it is not sufficient. Despite recent advances in the early detection and diagnosis of cervical cancer, the incidence is increasing and current treatments are unsatisfactory.

A number of studies have shown that a small proportion of tumor cells, designated as cancer stem cells, were capable of self-renewal and played a decisive role in tumor formation and growth $[3,4]$. Additionally, tumor recurrence after conventional therapy has been suggested to result from the presence of these slow-cycling cells, which are capable of initiating and sustaining neoplastic growth $[5,6]$. The resistance of cancer stem cells (CSCs) to conventional chemotherapy and radiotherapy has been attributed to cellular mechanisms such as multidrug resistance, quiescence, enhanced DNA repair abilities, and anti-apoptotic mechanisms [7]. Dysregulation of key signaling pathways such as the Wnt, Hedgehog, Notch, and TGF- $\beta$ /BMP pathways plays an important role in modulating tumorigenesis as well as in stem cell function [8]. These studies suggest that identification and targeting cancer stem cells are key aspects to optimizing cancer therapy. CSCs have been isolated and cultured as structured spheroids in the presence of serum and growth factors. Several markers have been proposed to identify and isolate cancer stem cells including CD133, CD44, CD24, CD90, CD34, CD117, CD20, and aldehyde dehydrogenase (ALDH1) [7, 9], a cytosolic enzyme that is responsible for the oxidation of retinaldehydes to retinoids $[10,11]$.

Some members of the ALDH super-family play key roles in the enzymatic detoxification of endogenous and exogenous aldehydes and in the formation of important 
metabolic molecules such as retinoic acid or gammaaminobutyric acid [12]. Thus, ALDH gene mutations lead to defective metabolism and provide the basic features of diseases such as gamma-hydroxybutyric aciduria, pyridoxinedependent seizures, Sjögren syndrome, or type II hyperprolinemia [13]. Recent studies have shown that ALDH1 is a cancer stem cell marker and that its presence strongly correlates with tumor malignancy and self-renewal properties of stem cells in different tumors, including breast cancer, hepatoma, and colon and lung cancer [14-17]. We have previously reported that expression of ALDH1 was found in $24.77 \%$ of cervical carcinoma by immunohistochemical staining. Additionally, flow cytometric analysis, qRT-PCR, and Western blot confirmed the presence of small subpopulations of ALDH1-positive cells. To examine the significance, we regulated and monitored ALDH1 gene expression by real time (RT)-PCR and Western blotting in HeLa cells. There was no change in the proliferation, apoptosis, and cell cycle after alteration of ALDH1 expression. Additionally, we demonstrated that reduction of ALDH1 led to markedly decreased migration, whereas restoration improved migration, suggesting that ALDH1 might potentially function as a marker of cervical CSC.

\section{Materials and methods}

\section{Cell culture}

The HeLa human cervical adenocarcinoma cell line was purchased from the American Type Culture Collection and maintained in RPMI 1640 medium (Life Technologies) supplemented with $10 \%$ fetal bovine serum (Invitrogen).

\section{SiRNA transfection}

Three small interfering RNAs (siRNAs) targeting specific sequences of ALDH1 (NM_017617) and a scrambled siRNA (not homologous to any gene) as a negative control were synthesized by GenePharma (Shanghai, China). Searches of the genome database (BLAST, SSEARCH) were conducted to ensure that these sequences would not target other genes. Preliminary experiments indicated that, of the three siRNAs, the siRNA targeting the specific sequence (sense'-CCAAAG UCCUGGAGGUUGAdTdT-3' and antisense 3'-dTdTGGU UUCAGGACCUCCAACU-5') yielded the greatest downregulation of ALDH1 expression (data not shown). This siRNA was selected for further investigation. A non-targeting siRNA (nt-siRNA), which did not target any known gene, was used as a negative control. We performed siRNA transfection efficiency assays using varying concentrations of siRNA and determined that $25 \mathrm{nmol}$ of siRNA provided optimal transfection efficiency (data not shown). In each experiment, the nt-siRNA transfection, particle stimulation-only groups (nontransfection, particle stimulation only), and the negative control $(\mathrm{NC}=$ nontransfection and nonparticle stimulation) groups were included as controls. HeLa cells were seeded into 6-well plates $\left(4-5 \times 10^{4}\right.$ cells/well) and cultured in $2 \mathrm{~mL}$ of basic culture medium containing $10 \%$ FBS until the cells were $70 \%$ confluent. The siRNA-Lipofectamine ${ }^{\mathrm{TM}} 2000$ complex was pre-mixed according to the manufacturer's instructions and added to the 6 -well plates. The results were confirmed by real-time RT-PCR.

\section{Plasmids and primers}

Human ALDH1 cDNA was cloned into the pIRES2-vector (Clontech, Santa Clara, CA) between the BamHI and the HindIII restriction sites (pIRES2/ALDH1), and a corrective construct was verified by DNA sequencing. The pIRES2/ ALDH1 construct was transfected into HeLa cells using Lipofectamine plus reagent (Invitrogen, San Diego, CA) following the manufacturer's instructions. The cells were cultured in the presence of $1 \mathrm{mg} / \mathrm{mL}$ of G418 to establish stable cell lines. The expression of ALDH1 was confirmed by Western blot and reverse transcriptase polymerase chain reaction.

\section{Quantitative real-time PCR}

All the reactions were performed in a $20-\mu \mathrm{L}$ reaction volume in triplicate by SYBR Green Real-time PCR Universal Reagent (GenePharma Co., Ltd.) and MX-3000P Real-time PCR equipment (Stratagen). Standard curves were generated,
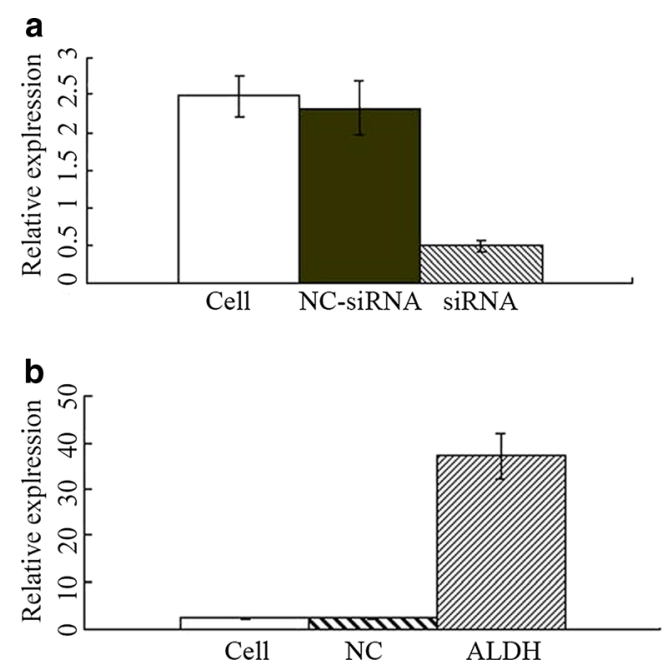

Fig. 1 Expression of $A L D H 1$ in various cells. a The mRNA expression of $A L D H 1$ in the cells without treatment, the cells with non-targeting siRNA and the cells with siRNA targeting the specific sequence. b The detection of $A L D H 1$ mRNA levels in cells without any treatment, the cells just with vector and cells with a pIRES2/ALDH1 construct 


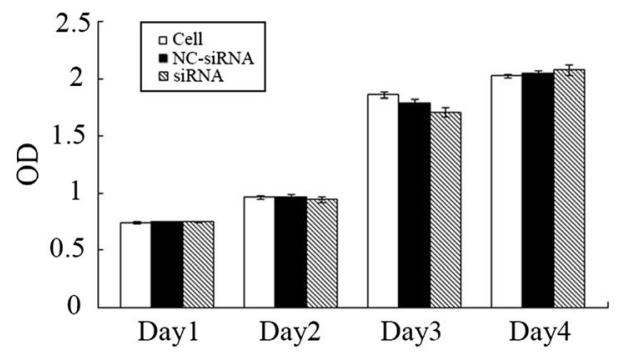

Fig. 2 The MTT assay to evaluate cell proliferation. a The OD value of the cells without any treatment, the cells with non-targeting of siRNA and the cells with siRNA targeting the specific sequence. $\mathbf{b}$ The OD value of

and the relative amount of ALDH1 was normalized to $18 \mathrm{~S}$ RNA $\left(2^{-\Delta \Delta \mathrm{Ct}}\right)$. The ALDH1 expression fold change was evaluated using $2^{-\Delta \Delta \mathrm{Ct}}$. The primers are ALDH1 (forward, 5'-CTGCTGGCGACAATGGAGT-3'; reverse, 5'-GTCAGC

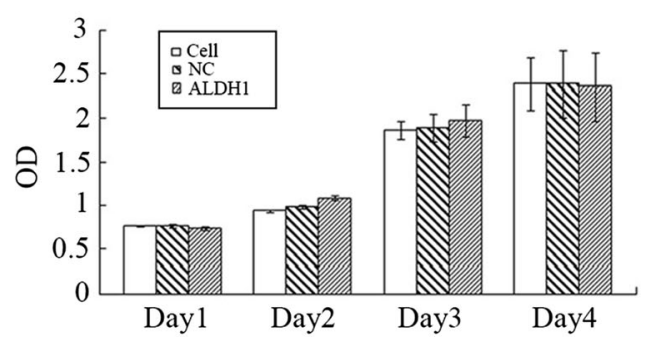

the cells without treatment, the cells with vector and the cells with a pIRES2/ALDH1 construct

CCAACCTGCACAG-3') and 18S rRNA (forward 5'-CCTG GATACCGCAGCTAGGA-3' and reverse 5'-GCGGCGCA ATACGAATGCCCC $-3^{\prime}$ ). These primers yielded 230- and 112-bp products, respectively.
Fig. 3 Cell death was monitored by Annexin V staining and flow cytometry. The right lower quadrant of each plot contains early apoptotic cells, whereas the right upper quadrant contains late apoptotic cells. This experiment was repeated on three independent occasions, and similar results were obtained each time. a The cells without any treatment, cells with nontargeting siRNA and cells with siRNA targeting the specific sequence. b The cells without any treatment, cells just with vector and cells with pIRES2/ALDH1 construct. c Each bar represents mean values \pm SE from three independent experiments for cells without any treatment, with nontargeting siRNA and with siRNA targeting the specific sequence. $\mathbf{d}$ Each bar represents mean values \pm SE from three independent experiments for cells without any treatment, cells just with vector and cells with pIRES2/ALDH1 construct
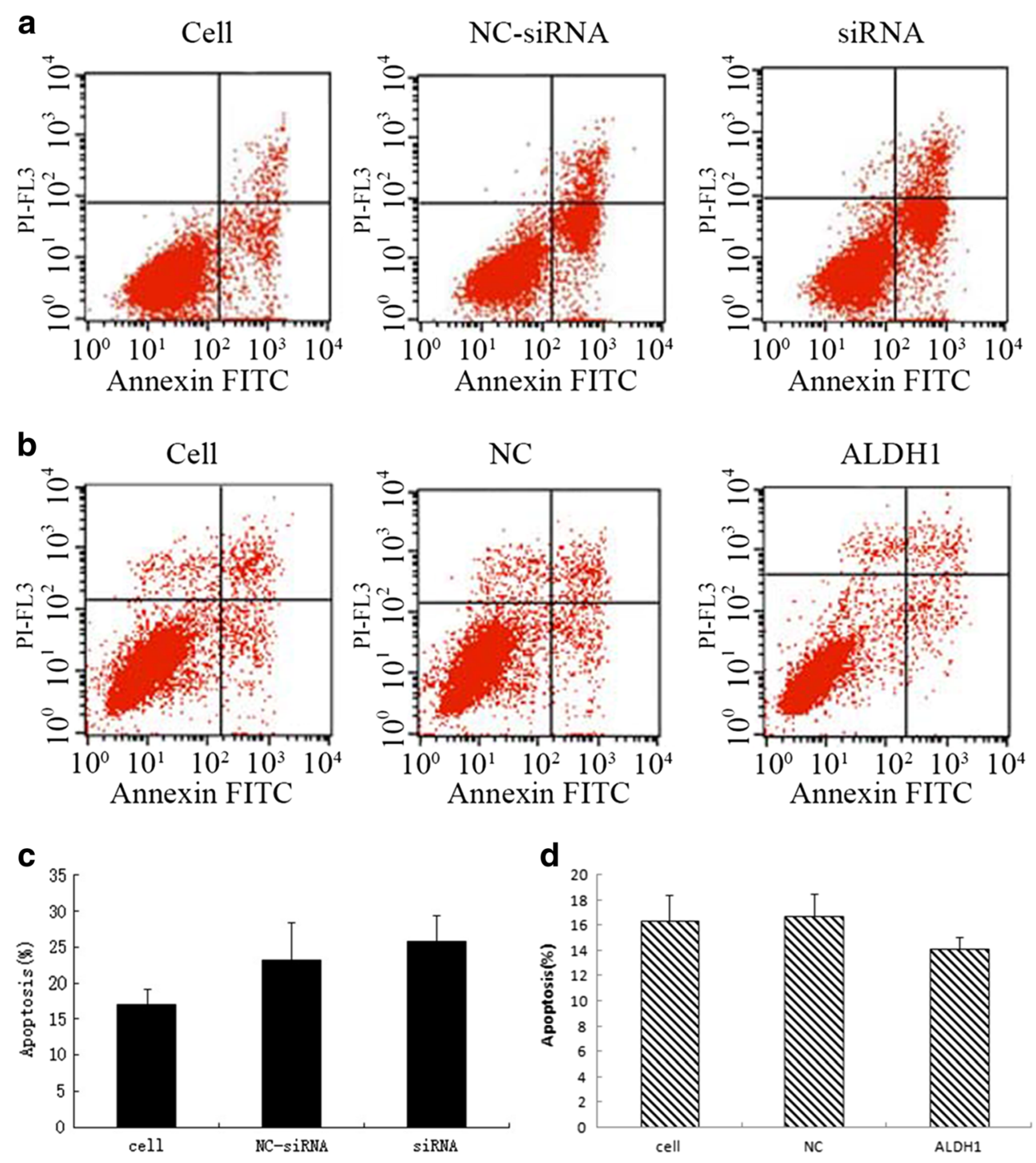

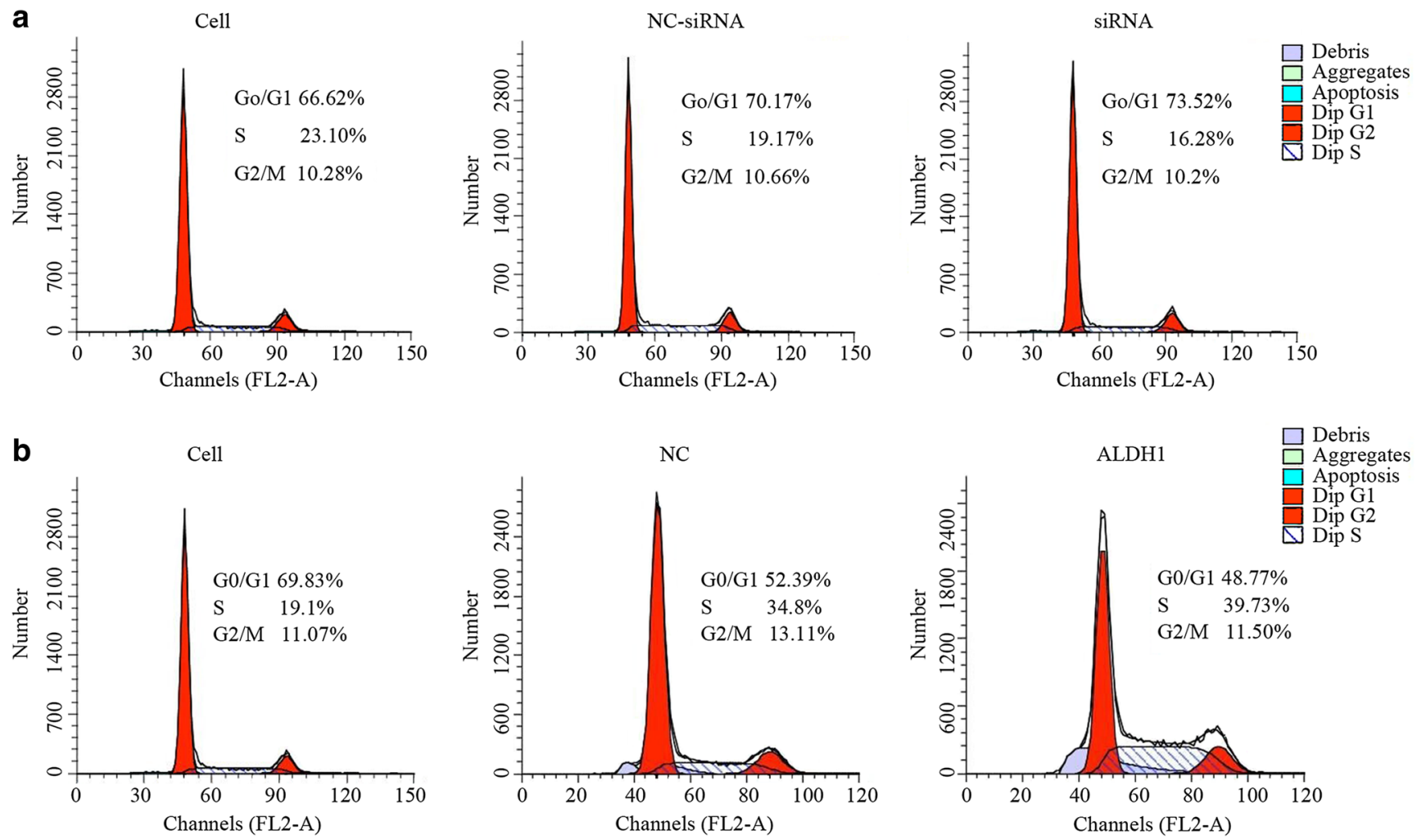

Fig. 4 The cell cycle distribution of the cells was analyzed by FACS. a The cells without any treatment, cells with non-targeting siRNA and cells with siRNA targeting the specific sequence. $\mathbf{b}$ The cells without any treatment, cells just with vector and cells with pIRES2/ALDH1 construct

\section{Cell proliferation assays}

The cell survival was assessed using the 3-(4,5-dimethylthiazol-2-yl)-5-(3-carboxymethoxyphenyl)-2(4-sulfophenyl)2H-tetrazolium (MTS) assay (Sigma). For the MTS assay (Promega), $1 \times 10^{4}$ cells/well were seeded in triplicate in a 96-well plate for each cell line. At 12, 24, and $48 \mathrm{~h}, 20 \mu \mathrm{L}$ of MTS reagent was added to each well and incubated for $1 \mathrm{~h}$ at $37^{\circ} \mathrm{C}$; the results were analyzed by a plate reader at $490 \mathrm{~nm}$. The sample data were normalized to the background readings of media only.

\section{Cell cycle analysis}

Forty-eight hours after transfection, the cells were harvested and fixed in $70 \%$ ice cold ethanol and followed by RNase A treatment, stained with $50 \mathrm{lg} / \mathrm{mL}$ of propidium iodide for the DNA content analysis by flow cytometry on a FACS Calibur system (EPICS ALTRA, Beckman Coulter, Fullerton, CA). The data were collected and processed using FlowJo FACS analysis software (Tree Star, Ashland, OR).

\section{Apoptosis assays}

The cell pellets were resuspended in Annexin V-FLUOS staining solution (Roche Molecular Biochemicals) and incubated for $15 \mathrm{~min}$ at room temperature. The samples were then analyzed on a FACS Calibur system (EPICS ALTRA, Beckman Coulter, Fullerton, CA).

\section{Transwell cell migration assay}

Forty-eight hours after transfection, the cells were resuspended in serum-free RPMI 1640 medium, and $5 \times 10^{4}$ cells were added to the upper chamber of Matrigel-coated 24-well plates ( 8 - $\mu \mathrm{m}$ pore size, Corning, Corning, NY). The lower compartment was filled with RPMI 1640 medium containing $20 \%$ FBS. After $24 \mathrm{~h}$, the cells that had not migrated were removed from the upper face of the filters using cotton swabs, and the cells that had migrated to the lower surface of the filters.

\section{Statistical analyses}

The normally distributed continuous variables were compared by a one-way analysis of variance (ANOVA). When significance between the groups was apparent, multiple comparisons of the means were performed using the Bonferroni procedure with type I error adjustment. The data are presented as the means \pm standard deviation (SD). $P$ values of less than 0.05 were deemed significant. Repeated measurements with a linear mixed model were conducted to determine the group effect in the change of the MTS OD values among the groups. The statistical 

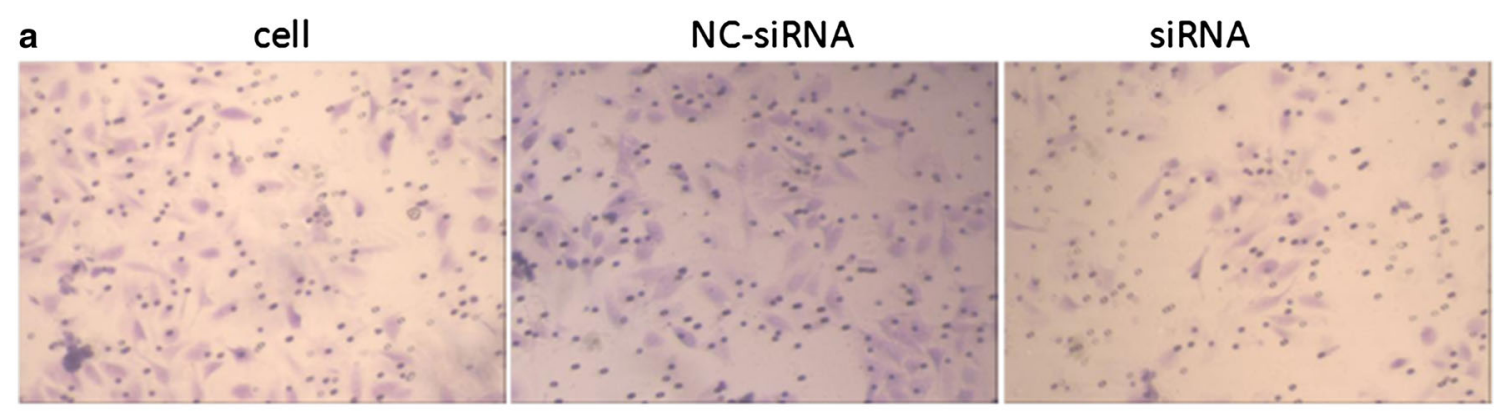

b

NC

ALDH1
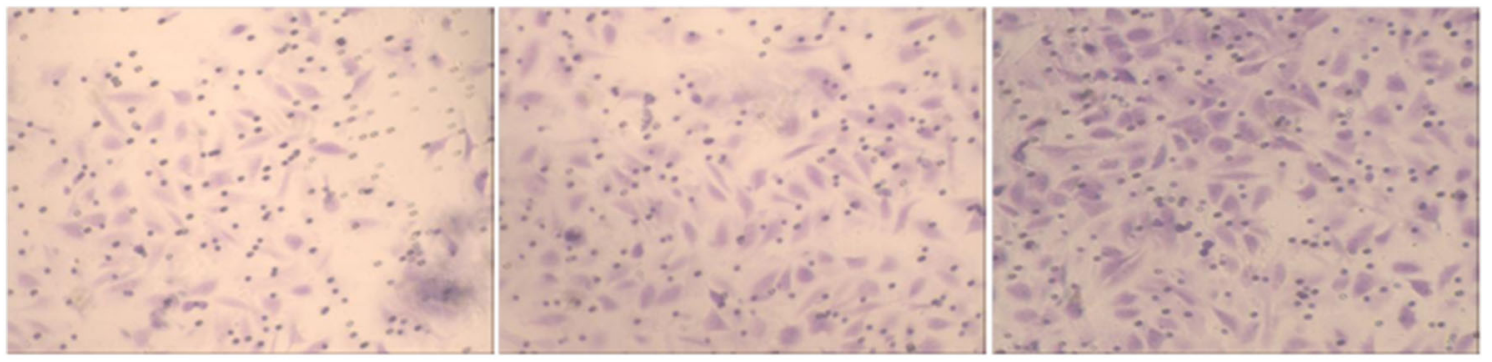

c

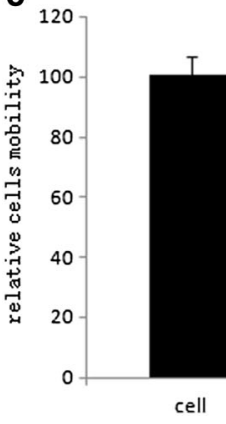

cell
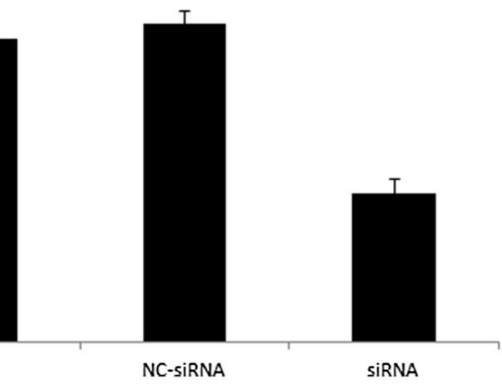

SiRNA

Fig. 5 The Transwell analysis of migration. a The cells without any treatment, cells with non-targeting siRNA and cells with siRNA targeting the specific sequence. b The cells without any treatment, cells just with vector and cells with pIRES2/ALDH1 construct. c Each bar represents mean values $\pm \mathrm{SE}$ from three independent experiments for

assessments were two-sided and evaluated at the 0.05 level of significant difference. The statistical analyses were performed using SPSS 15.0 statistical software (SPSS, Inc., Chicago, IL).

\section{Results}

\section{ALDH1 gene expression in HeLa cells was successfully disturbed by transfecting siRNA or overexpressing plasmid}

RNAi technology was employed to silence the ALDH1 expression. Real-time PCR and Western blot were performed to quantify ALDH1 expression. Endogenous ALDH1 mRNA expression (Fig. 1a) were significantly lowered by RNAi (HeLa-ALDH1-RNAi) than by the control transfections (HeLa-NCi) and HeLa cells. To increase the ALDH1

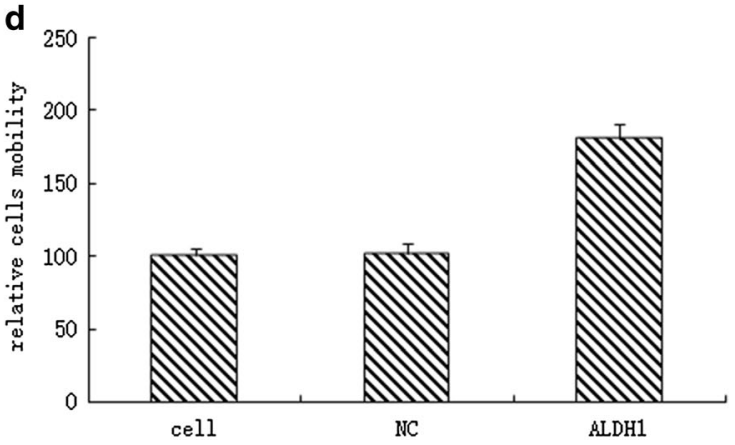

cells without any treatment, with non-targeting siRNA and with siRNA targeting the specific sequence. d Each bar represents mean values $\pm \mathrm{SE}$ from three independent experiments for cells without any treatment, cells just with vector and cells with pIRES2/ALDH1 construct

expression, the cells were stably transfected with an ALDH1 expression construct. After transfection and G418 selection, the ALDH1 mRNA (Fig. 1b) expression levels in the stably transfected cells (HeLa-ALDH1) were much higher than in the HeLa cells.

\section{No effect of ALDH1 on proliferation, apoptosis, and cell cycle}

As shown in Fig. 2a, knockdown of ALDH1 using the oligonuclear sequences resulted in no significant decrease and no significant increase in the HeLa cell proliferation (Fig. 2a), apoptosis (Fig. 3a, c), and cell cycle (Fig. 3a) compared to the empty vector-transfected cells. Similarly, no obvious alteration of the HeLa cells was found in the ALDH1 overexpressing cells in comparison to that in the control sequence-transfected group (Figs. 2b, 3b, c, and 4b). 


\section{ALDH1 influences HeLa cell migration in vitro}

To examine the effect of ALDH1 on the mobility of HeLa cells, the migration was measured by Transwell assays. We showed that the SiRNA-transfected HeLa cells had lower migration rates than the control (Fig. 5a, c), whereas the HeLaALDH1-positive cells had significantly higher migration rates than their control cells (Fig. 5b, d), suggesting that ALDH1 is an effective target for treating cervical cancer metastases.

\section{Discussion}

Recurrence and metastasis are the most important factors affecting the prognosis of patients with cervical cancer [18]. Developing molecular biomarkers to detect cancer, predicting disease progression, and monitoring therapeutic response would be beneficial. ALDH1 is a detoxifying enzyme responsible for the oxidation of retinol to retinoic acid, and it has a role in the early differentiation of stem cells.

Recent studies showed that ALDH1 expression was associated with tumor cell metastasis in vivo $[19,20]$. According to the report of the Sidney Kimmel Comprehensive Cancer Center in the USA, a high ALDH expression is associated with worse overall survival in patients who have undergone resection for early-stage disease. The European Society for Medical Oncology (ESMO) has established Practice Guidelines that suggest that enhanced clonogenic growth of ALDH+ cancer cells has an important role in the long-term outcome of patients diagnosed with pancreatic adenocarcinoma by mediating or even stimulating cancer dissemination throughout the abdominal cavity and resistance to chemotherapy with 5-fluorouracil (5-FU) or gemcitabine [21].

To confirm whether ALDH1 was involved in cervical cancer metastasis, we performed a Transwell migration assay in vitro to investigate the effect of ALDH1 on HeLa cell mobility. Similar to those of previous studies, our results showed that the migrated rate of cells was concordant with ALDH1 expression. However, our results indicated that ALDH1 has no relationship with cell proliferation, which was not consistent with previous reports. Additionally, we found that the rates of apoptotic cells and cell cycle distribution did not present any significant alteration. The finding suggested that ALDH1 might participate in the tumorigenesis of cervical cancer through pathways other than the promotion of cell growth and obstruction of apoptosis. ALDH1 might be involved in metastasis in cervical cancer patients. ALDH1 could be used as a therapeutic target.

\section{Conclusion}

Our results demonstrated that ALDH1 might be involved in cervical cancer metastasis. Further experiments in vivo are necessary to clarify that ALDH1 might be a promising target and prognostic predictor for treating cervical cancer metastases.

Acknowledgments This work was supported by the National Natural Science Foundation of China (81101979 and 30872743), the Guangdong Province Natural Scientific Grant (S2011040004639 and S2012020010913), the Guangdong Province Medical Science Technology Grant (B2011088), the Key Clinical Program of the Ministry of Health ([2010] 439), the Guangdong College Students' Innovation and Entrepreneurship Training Program (1055813194), the National College Students' Innovation and Entrepreneurship Training Program (201310558097), the PhD Program Foundation of the China Education Ministry (20120171110097), the Yat-sen Scholarship for Young Scientists, and the Young Scientists Program Training Project at Sun Yat-sen University (11ykpy31).

\section{Conflicts of interest None}

Open Access This article is distributed under the terms of the Creative Commons Attribution 4.0 International License (http:// creativecommons.org/licenses/by/4.0/), which permits unrestricted use, distribution, and reproduction in any medium, provided you give appropriate credit to the original author(s) and the source, provide a link to the Creative Commons license, and indicate if changes were made.

\section{References}

1. Yao T, Chen Q, Zhang B, Zhou H, Lin Z. The expression of ALDH1 in cervical carcinoma. Med Sci Monit. 2011;17(8): HY21-26.

2. Stanley M. Pathology and epidemiology of HPV infection in females. Gynecol Oncol. 2010;117(2 Suppl):S5-10.

3. Lorico A, Rappa G. Phenotypic heterogeneity of breast cancer stem cells. J Oncol. 2011;2011:135039.

4. Reya T, Morrison SJ, Clarke MF, Weissman IL. Stem cells, cancer, and cancer stem cells. Nature. 2001;414(6859):105-11.

5. Sagar J, Chaib B, Sales K, Winslet M, Seifalian A. Role of stem cells in cancer therapy and cancer stem cells: a review. Cancer Cell Int. 2007;7:9.

6. Ghaffari S. Cancer, stem cells and cancer stem cells: old ideas, new developments. F1000 Med Rep. 2011;3:23.

7. Saini V, Shoemaker RH. Potential for therapeutic targeting of tumor stem cells. Cancer Sci. 2010;101(1):16-21.

8. Chang CC, Bredeson C, Juckett M, Logan B, Keever-Taylor CA. Tumor load in patients with follicular lymphoma post stem cell transplantation may correlate with clinical course. Bone Marrow Transplant. 2003;32(3):287-91.

9. Friedman GK, Gillespie GY. Cancer stem cells and pediatric solid tumors. Cancers (Basel). 2011;3(1):298-318.

10. Wirth T, Schmuck K, Tietze LF, Sieber SA. Duocarmycin analogues target aldehyde dehydrogenase 1 in lung cancer cells. Angew Chem Int Ed Engl. 2012;51(12):2874-7.

11. Crandall JE, Goodman T, McCarthy DM, Duester G, Bhide PG, Dräger UC, et al. Retinoic acid influences neuronal migration from 
the ganglionic eminence to the cerebral cortex. J Neurochem. 2012;119(4):723-35.

12. Jackson B, Brocker C, Thompson DC, Black W, Vasiliou K, Nebert DW, et al. Update on the aldehyde dehydrogenase gene (ALDH) superfamily. Hum Genom. 2011;5(4):283-303.

13. Ginestier C, Hur MH, Charafe-Jauffret E, Monville F, Dutcher J, Brown M, et al. ALDH1 is a marker of normal and malignant human mammary stem cells and a predictor of poor clinical outcome. Cell Stem Cell. 2007;1(5):555-67.

14. Ma S, Chan KW, Lee TK, Tang KH, Wo JY, Zheng BJ, et al. Aldehyde dehydrogenase discriminates the CD133 liver cancer stem cell populations. Mol Cancer Res. 2008;6(7):1146-53.

15. Huang EH, Hynes MJ, Zhang T, Ginestier C, Dontu G, Appelman $\mathrm{H}$, et al. Aldehyde dehydrogenase 1 is a marker for normal and malignant human colonic stem cells (SC) and tracks SC overpopulation during colon tumorigenesis. Cancer Res. 2009;69(8):3382-9.

16. Jiang F, Qiu Q, Khanna A, Todd NW, Deepak J, Xing L, et al. Aldehyde dehydrogenase 1 is a tumor stem cell-associated marker in lung cancer. Mol Cancer Res. 2009;7(3):330-8.
17. Stanley M. Prophylactic human papillomavirus vaccines: will they do their job? J Intern Med. 2010;267(3):251-9.

18. Morimoto K, Kim SJ, Tanei T, Shimazu K, Tanji Y, Taguchi T, et al. Stem cell marker aldehyde dehydrogenase 1-positive breast cancers are characterized by negative estrogen receptor, positive human epidermal growth factor receptor type 2, and high Ki67 expression. Cancer Sci. 2009;100(6):1062-8.

19. Wakamatsu Y, Sakamoto N, Oo HZ, Naito Y, Uraoka N, Anami K, et al. Expression of cancer stem cell markers ALDH1, CD44 and CD133 in primary tumor and lymph node metastasis of gastric cancer. Pathol Int. 2012;62(2):112-9.

20. Charafe-Jauffret E, Ginestier C, Iovino F, Tarpin C, Diebel M, Esterni B, et al. Aldehyde dehydrogenase 1-positive cancer stem cells mediate metastasis and poor clinical outcome in inflammatory breast cancer. Clin Cancer Res. 2010;16(1):45-55.

21. Cascinu S, Falconi M, Valentini V, Jelic S. ESMO guidelines working group. pancreatic cancer: ESMO clinical practice guidelines for diagnosis, treatment and follow-up. Ann Oncol. 2010;21 Suppl 5: v55-58. 